\title{
Città del Vaticano, Biblioteca Apostolica Vaticana, Vat. Pal. gr. 174
}

\author{
I. [Costantinopoli], fine XIII; II. e III. Costantinopoli, XIV.
}

Comp.; ff. I-III, 1-302, IV; num. moderna vergata in inchiostro nero nel marg. sup. est.; i ff. 135, 277 presentano un'ulteriore numerazione a matita; sul f. II in alto vi è il numero della capsa in cui il codice era conservato, $C$ 85, seguito dall'indicazione della provenienza dalla Biblioteca Palatina di Heidelberg: sum de Bibliotheca, quam Heidelberga capta Spolium fecit \& P. M. Gregorio XV trophaeum misit. Maximilianus utriusque Bavariae Dux \& c. S.R.I. Archidapifer et Princeps Elector; in basso, sullo stesso foglio, è indicato l'anno in cui la Biblioteca Palatina confluì nella Biblioteca Vaticana: Anno Christi 1623; sul f. III, grazie ad un'operazione di restauro, è stato recuperato il pinax vergato da Isacco Argiro, preceduto in alto dalla scritta latina carte 302, in hoc volumine continentur infrascripti luciani sermones. Legatura in pelle bianca con segnatura crisografa sul dorso.

Questo testimone dei Dialoghi di Luciano consta di due unità codicologiche: quella più antica (ff. 1-55, 57-120) risale allo scorcio del XIII secolo, quella più recente (f. 56, ff. 120-302), vergata da Isacco Argiro, si deve riferire al terzo quarto del XIV secolo. La tipologia degli interventi eseguiti da Argiro nel codice consente di ipotizzare che la riunione in un unico volume sia legata a sue esigenze di studio e alla necessità di possedere un testimone corretto contenente l'intera opera lucianea.

\section{I. ff. 1-55, 57-120 (Costantinopoli ?, fine XIII sec.)}

Cart. or.; $1-6^{8}(48), 7^{7}$ (55), $8-15^{8}(120)$; num. fasc. sul marg. inf. int. del recto del primo foglio di ogni fasc. e sul marg. inf. int. del verso dell'ultimo foglio di ogni fasc.; mm $250 \times 169=37$ // 175 // $47 \times 17$ // 120 // 30; a piena pagina, ll. 31-32, unità di rigatura mm 6 (f. 24r); non vi è alcuna traccia di rigatura.

\section{Scrittura}

Si individua un solo copista: mano A (ff. 1r-55v, 57r-120v) esibisce una scrittura d'erudito di modulo piccolo, vergata con ductus particolarmente corsivo, in cui si riscontra un uso elevato di segni tachigrafici e abbreviazioni, riferibile allo scorcio del secolo XIII. 


\section{Decorazione}

La decorazione non originaria è stata eseguita da <Isacco Argiro>, il quale ha vergato in rosso carminio le iniziali minori poste in ekthesis e leggermente ingrandite e i numeri di ciascun opuscolo nel margine esterno.

\section{Interventi successivi}

Argiro ha aggiunto, nell'interlinea (cf. ff. 7v, 10r, 10v, 11r) o nei margini (cf. f. 16v) a seconda della loro lunghezza, porzioni testuali omesse dal copista principale. Al primo copista si devono invece le invocazioni poste nei margini dei ff. 1, 15, 75, 99.

\section{Contenuto}

Luciano di Samosata, Orationes cum scholiis (ff. 1-2r, 1. 20, op. 3, Hippias seu Balneum, ed. Bompaire 1993 I, 35-41; ff. 2r-6r, 1. 22, op. 10, De domo, ed. Bompaire 1993 I, 155172; ff. 6r-7v, 1. 6 a. i., op. 7, Muscae encomium, ed. Bompaire 1993 I, 79-86; ff. 7v, 1.5 a. i.-11v, 1. 10 a. i., op. 9, Demonactis vita, ed. Bompaire 1993 I, 126-145; ff. 11v-16v, op. 8, Nigrinos, ed. Bompaire 1993 I, 96-117; ff. 17r-22r, 1. 10, opp. 1-2, Phalaris A et B, ed. Bompaire 1993 I, 8-29; ff. 22r-23v, 1. 4 a. i., opp. 5-6, Hercules-De electro seu Cynis, ed. Bompaire 1993 I, 59-73; ff. 23r-25v, op. 11, Patriae encomium, ed. Bompaire 1998, II, 1-13); Pseudo-Luciano, Macrobii longaevi (ff. 25r-27v, ed. Bompaire 1998 II, 24-37); Luciano di Samosata, Orationes (ff. 28r-47r, Verae Historiae I et II - Calumniae non temere credendum, ed. Bompaire 1998 II, 56-168; ff. 47r, 1. 3-53r, op. 17, Symposium seu convivium seu Lapithae, ed. Bompaire 1998 II, 200-230; ff. 53r-55v e 57r-58r, op.

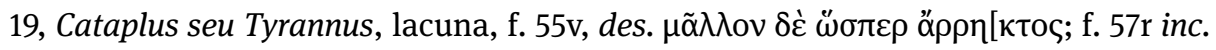

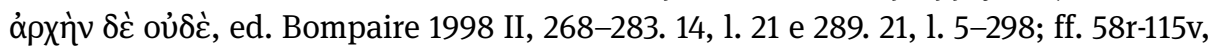
opp. 20-29, Iuppiter confutatus - Iuppiter tragoedus - Gallus - Prometheus seu Caucasus - Icaromenippus - Timon seu misanthropos - Charon s. contemplantes - Vitarum Auctio - Piscator seu reviviscentes - Bis accusatus seu tribunalia, ed. Bompaire 1998 II, 312-328-III, 2003, 18-333-IV, 2008, 16-253; ff. 115v-117v, op. 30, De sacrificiis, ed. Iacobitz 1888 I, 222-229; ff. 117v-120v, op. 31, Adversus Indoctum et libros multos ementem,

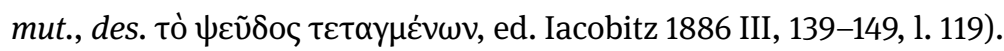

\section{II. f. 56 restauro (Costantinopoli, terzo quarto del XIV sec.)}

Cart.; fil. non visibile; foglio aggiunto al fasc. 7 (supra); mm $251 \times 171=36$ // 168 // $47 \times 20$ // 118 // 35; a piena pagina, 11. 30, unità di rigatura mm 6; sul recto del foglio sono tracciate unicamente le linee di giustificazione atte a delimitare lo spazio scrittorio. 


\section{Scrittura}

Si individua un solo copista: <Isacco Argiro> (f. 56rv).

\section{Contenuto}

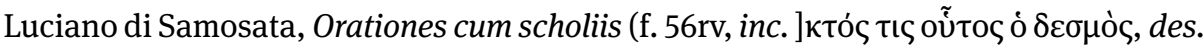

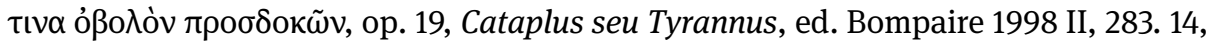
1. 21-289. 21, 1. 5).

\section{III. ff. 120-302 (Costantinopoli, terzo quarto del XIV sec.)}

Cart.; fil. ff. 121, 124, 126, 129, 132, 134, 135, 140, 141, 142, 143 simile a cloche (M/Tr nr. 3008, 1358; Br nr. 4019-4020, 1360), ff. 144 145, 146, 148, 153, 156, 157, 158, 160, 163, 166, 167, 170, 172, 182, 184, 186, 187, 189, 191, 196, 197, 202, 203, 204, 206, 207, 209, 210, 211, 212, 216, 217, 221, 222, 223, 230, 231, 232, 233, 240, 241, 242, 243, 246, 251, 252, 256, 257, 262 simile a poire (M/Tr nr. 4354, 1361; Br nr. 7376, 1355-1356), ff. 266, 267, 268, 271, 272, 274, 277, 279, 281, 283, 285, 287, 288, 289, 294, 296, 298 simile a arbalète (M/Tr nr. 250, 1356; Br nr. 730, 1326); 16-17 $(136), 18^{6+1}(143), 19^{8}(151), 20-30^{10}(261), 31-34^{8}$ (293), $35^{8+1}$ (302); num. dei fascicoli presente nel marg. inf. est. sul recto del primo foglio di ogni fascicolo e sul marg. inf. est. del verso dell'ultimo foglio di ogni fascicolo; mm $252 \times 170=35 / / 170$ // $49 \times 17$ // 117 // 36; a piena pagina, ll. 34-36 (ff. 144-302); ll. 30 (ff. 121-143), unità di rigatura pari a $5 \mathrm{~mm}$; resta traccia sul recto dei fogli di una rigatura atta ad inquadrare lo spazio scrittorio.

\section{Scrittura}

Si individua un solo copista: <Isacco Argiro (Menchelli 2014, 184)> (ff. 121r-302v).

\section{Decorazione}

Decorazione minima: Argiro ha vergato in rosso carminio unicamente le iniziali minori e i numerali relativi agli opuscoli.

\section{Interventi successivi}

Si devono ad Argiro alcune note a margine per segnalare integrazioni testuali o informazioni circa la trasmissione di alcuni opuscoli; in merito all'Alcione, al f. 269v Argiro

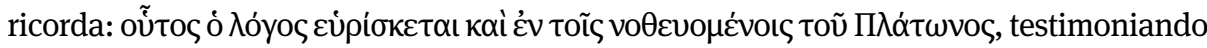
una doppia trasmissione medievale del trattato, nel corpus platonico e in quello lucianeo. 


\section{Contenuto}

Luciano di Samosata, Orationes cum scholiis (ff. 121-122, 1. 6, fine di op. 31, Adversus Indoctum et libros multos ementem, ed. Iacobitz 1887 III, 149, 119-153; ff. 123v-132r, 1.5 a.i., op. 36, De mercede conductis, ed. Iacobitz 1888 I, 292-319; ff. 132r-135r, op. 65, Apologia, ed. Iacobitz 1888 I, 319-327; ff. 135r-136v, op. 16, Iudicium vocalium, ed. Iacobitz 1888 I, 28-33); Pseudo-Luciano, Soloecista seu pseudosophista (ff. 136v-139r, op. 18, ed. Bompaire 1998 II, 242-256); ff. 139r-147r, op. 33, De parasito seu artem esse parasiticam, ed. Iacobitz 1887 III, 25-52); Luciano di Samosata, Orationes (ff. 147r-150v, op. 56, Fugitivi, ed. Iacobitz 1887 III, 287-301; ff. 151r-157r, op. 43-50, Imagines - Pro imaginibus, ed. Iacobitz 1883 II, 283-265; ff. 157r-166v, op. 57, Toxaris seu amicitia, ed. Iacobitz 1883 II, 265-302; ff. 166v-168v, op. 68, Scytha, ed. Iacobitz 1888 I, 404-410; ff. 168v-171v, op. 38, Menippus seu Necromantia, ed. Iacobitz 1888 I, 189-204; ff. 171v-173r, op. 40, De luctu, ed. Iacobitz 1887 III, 77-84; ff. 173r-177v, op. 54, Abdicatus, ed. Iacobitz 1883 II, 85-103; ff. 177v-183r, op. 73, Navigium seu vota, ed. Iacobitz 1883 II, 213-234; ff. 183v-184r, op. 62, Herodotus, ed. Iacobitz 1888 I, 391-394; ff. 184r-185v, op. 63, Zeuxis, ed. Iacobitz 1888 I, 394-400; ff. 186r-187v, op. 64, Pro lapsus inter salutandum, ed. Iacobitz 1888 I, 328-334; ff. 187v188v, op. 47, Eunuchus, ed. Iacobitz 1883 II, 182-187; ff. 188v-189v, op. 67, Disputatio cum Hesiodo, ed. Iacobitz 1887 III, 209-213; ff. 189v-190v, op. 60, Dipsades, ed. Iacobitz 1887 III, 205-209; ff. 190v-193r, op. 53, Tyrannicida, ed. Iacobitz 1883 II, 74-85; ff. 193r-194r, op. 66, Harmonides, ed. Iacobitz 1888 II, 400-404; ff. 194r-201v, op. 59, Quomodo historia conscribenda sit, ed. Iacobitz 1883 II, 1-30; ff. 201v-205v, op. 55, De morte Peregrini, ed. Iacobitz 1887 III, 271-287; ff. 205v-209r, Pseudologista seu de die nefasto, ed. Iacobitz 1887 III, 166-181; ff. 209r-221v, op. 70, Hermotimus seu de sectis, ed. Iacobitz 1888 I, 334-390; ff. 222r-224v, op. 46, Lexiphanes, ed. Iacobitz 1883 II, 171-182; ff. 224v-228r, op. 41, Rhetorum praeceptor, ed. Iacobitz 1887 III, 84-97; ff. 228r-234r, op. 37, Anacharsis, ed. Iacobitz 1887 III, 52-77); Pseudo-Luciano, De saltatione (ff. 234r-240r, op. 45, ed. Iacobitz 1883 II, 143170); Libanio, Orationes (ff. 240v-249v, op. 64, De Saltatoribus (Libanii ad Aristidem), ed. Foerster 1908 IV, 420-498); Luciano di Samosata, Orationes (ff. 249v-250v, op. 71, Prometheus es in verbis, ed. Iacobitz 1888 I, 8-12); Pseudo-Luciano, Amores (ff. 250v-257v, op. 49, ed. Iacobitz 1888 I, 207-238); Luciano di Samosata, Orationes (ff. 257v-260r, op. 61, Saturnalia, ed. Iacobitz 1887 III, 301-311; ff. 260r-262v, Epistulae Saturnalia, ed. Iacobitz 1887 III, 311-322; ff. 262v-264r, op. 52, Deorum concilium, ed. Iacobitz 1887 III, 385-392; ff. 264r-269v, op. 34, Philopseudeus seu incredulus, ed. Iacobitz 1887 III, 97-121); Platone, Halcyon seu de transformatione (ff. 269v-270v, ed. Iacobitz 1888 I, 58-62); Luciano di Samosata, Alexander sive Pseudomantis (ff. 270v-277v, ed. Iacobitz 1883 II, 168-208); Pseudo-Luciano, Demosthenis Encomium (ff. 277v-285r, ed. Iacobitz 1887 III, 374-384); Nero (ff. 285r-286r, ed. Iacobitz 1887 III, 439-443); Luciano di Samosata, Orationes (ff. 286r-287v, op. 32, Somnium seu vita Luciani, ed. Iacobitz 1888 I, 1-8; ff. 287v-297r, op. 39, Asinus seu Lucius, ed. Iacobitz 1883 II, 303-338); Pseudo-Luciano, De astrologia (ff. 295v297r, op. 48, ed. Iacobitz 1883 II, 187-195); De Syria Dea (ff. 297r-302v, op. 44, mut. alla

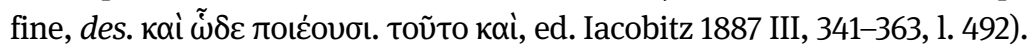




\section{Relazioni stemmatiche}

Il Pal. gr. $174(B)$ si divide tra due famiglie della tradizione manoscritta dei dialoghi di Luciano: per il testo recato nell'unità più antica il codice appartiene alla classe ( $y)$; per quello della parte più recente afferisce alla classe $\beta$ ed è vicino al Vat. gr. $87(A)$ e al Par. gr. 2957 (N). Secondo l'editore, Argiro ha corretto il testo vergato dal primo copista servendosi di un codice legato a T (Vat. Pal. gr. 213), della famiglia L e molto contaminato da $\beta$ (ed. Bompaire 1993, I, CI). Sembra verosimile supporre che Argiro abbia adoperato il medesimo esemplare sia per supplire il codice nella seconda unità sia per correggere e rivedere il testo del primo blocco.

\section{Storia del codice}

Il codice è presente nella Biblioteca Apostolica Vaticana dal 1625, anno in cui i libri della Biblioteca Palatina di Heidelberg lasciati in dono a Gregorio XV (1621-1623) confluirono in quella papale (Odier 1973, 107-108). In un inventario che tiene conto di queste recenti acquisizioni si fa menzione del fatto che il codice Vat. Pal. gr. 174 è appartenuto a Giannozzo Manetti (olim Ianotti Manetti) (Stevenson 1885, 91).

\section{Bibliografia}

\section{Cataloghi}

Odier (1973) 107-108; Stevenson (1885) 91-92.

\section{Edizioni}

Bompaire (1993-2008); Foerster (1908); lacobitz (1883-1888).

\section{Codicologia e paleografia}

Menchelli (2014) 184.

\section{Riproduzioni}

Menchelli (2014) tav. 2. 